\title{
Hard X-ray emission from a young massive star-forming cluster ${ }^{\star}$
}

\author{
H. Beuther ${ }^{1}$, J. Kerp ${ }^{2}$, T. Preibisch ${ }^{1}$, T. Stanke ${ }^{1}$, and P. Schilke ${ }^{1}$ \\ 1 Max-Planck-Institut für Radioastronomie, Auf dem Hügel 69, 53121 Bonn, Germany \\ 2 Radioastronomisches Institut der Universität Bonn, Auf dem Hügel 71, 53121 Bonn, Germany
}

Received 18 June 2002 / Accepted 27 August 2002

\begin{abstract}
We report the detection of hard X-ray emission $(>2 \mathrm{keV})$ from a number of point sources associated with the very young massive star-forming region IRAS $19410+2336$. The X-ray emission is detected from several sources located around the central and most deeply embedded mm continuum source, which remains undetected in the X-ray regime. All X-ray sources have $K$-band counterparts, and those likely belonging to the evolving massive cluster show near-infrared colors in the 2MASS data indicative of pre-main-sequence stages. The X-ray luminosities around $10^{31} \mathrm{erg} \mathrm{s}^{-1}$ are at the upper end of luminosities known for low-mass pre-main-sequence sources, and mass estimates based on the infrared data indicate that at least some of the $\mathrm{X}$-ray detected sources are intermediate-mass objects. Therefore, we conclude that the X-ray emission is due to intermediatemass pre-main-sequence Herbig Ae/Be stars or their precursors. The emission process is possibly due to magnetic star-disk interaction as proposed for their low-mass counterparts.
\end{abstract}

Key words. accretion, accretion disks - stars: early type - stars: formation - ISM: dust, extinction radiation mechanisms: thermal

\section{Introduction}

In the past, star formation research in the X-ray regime has focused strongly on low-mass objects, e.g., T Tauri stars. These objects emit mainly in the soft range of the X-ray spectrum $(<2 \mathrm{keV})$ with typical X-ray luminosities between $10^{28}-10^{30} \mathrm{erg} \mathrm{s}^{-1}$. The satellite observatory ROSAT was an ideal instrument to study such stars within a few 100 pc distance from the Sun, and the observed emission can be explained by enhanced solar-type magnetic activity (Feigelson $\&$ Montmerle 1999). In the last few years, a rising number of Class I protostars, which are still deeply embedded within their natal molecular cores $\left(A_{\mathrm{V}} \approx 10-100 \mathrm{mag}\right)$, have been detected in the hard X-ray regime between 2 and $10 \mathrm{keV}$ with X-ray luminosities higher than $10^{30} \mathrm{erg} \mathrm{s}^{-1}$. These detections were mostly made with the X-ray satellites ASCA and, most recently, with Chandra. Magnetic star-disk interactions are thought to be the most likely explanation for the hard X-ray emission (Hayashi et al. 1996; Montmerle et al. 2000). Furthermore, X-ray variability is observed in all types of low-mass pre-main-sequence objects. For an excellent

Send offprint requests to: $\mathrm{H}$. Beuther,

e-mail: beuther@mpifr-bonn.mpg.de

* Based on observations with the Chandra X-ray observatory, the Calar Alto $3.5 \mathrm{~m}$ telescope and the IRAM Plateau de Bure Interferometer. Chandra is operated by the NASA and the CXC, Calar Alto by the Max-Planck-Institut for Astronomy in Heidelberg, jointly with the Spanish National Commission for Astronomy, and IRAM is supported by INSU/CNRS (France, MPG (Germany), and IGN (Spain). recent review on these topics see Feigelson \& Montmerle (1999). Recently, Tsuboi et al. (2001) and Tsujimoto et al. (2002) reported the first tentative detections of deeply embedded Class 0 protostellar candidates in OMC3 by Chandra.

In comparison with the low-mass regime, X-ray observations of massive star-forming regions have been rare. Due to the high visual extinction within such regions $\left(A_{\mathrm{v}}\right.$ up to a few 100 or even 1000), soft X-ray emission is completely absorbed by the gas along the line of sight. Hofner \& Churchwell (1997) detected with ASCA for the first time hard X-ray emission in the massive star-forming region W3. Because of the low angular resolution of ASCA $\left(>1^{\prime}\right)$ they could not determine whether the emission is caused by the superposition of many point sources, e.g., protostellar clusters, or whether it is due to a wind-shocked cavity resulting from strong stellar winds interacting with the surrounding medium. Recently, Churchwell (2001) reported that Chandra data of the same region with a spatial resolution of $0.5^{\prime \prime}$ resolve the emission into many individual sources distributed over the entire W3 complex. Similarly, Garmire et al. (2000) and Feigelson et al. (2002) reported about 1000 X-ray emitting pre-main-sequence stars between 0.05 and $50 M_{\odot}$ in the Orion Nebula. Additionally, Zinnecker \& Preibisch (1994) found X-ray emission with the ROSAT satellite in the soft $\mathrm{X}$-ray band associated with several intermediate-mass Herbig Ae/Be pre-main-sequence stars. The derived X-ray luminosities for the Herbig Ae/Be stars are ranging between $10^{30} \mathrm{erg} \mathrm{s}^{-1}$ and $10^{32} \mathrm{erg} \mathrm{s}^{-1}$. Preibisch \& Zinnecker (1995) speculated that the emission might originate from coronal activity due to shear dynamo action. A study of the more distant molecular clouds Monoceros and Rosette also found indirect evidence for X-ray 

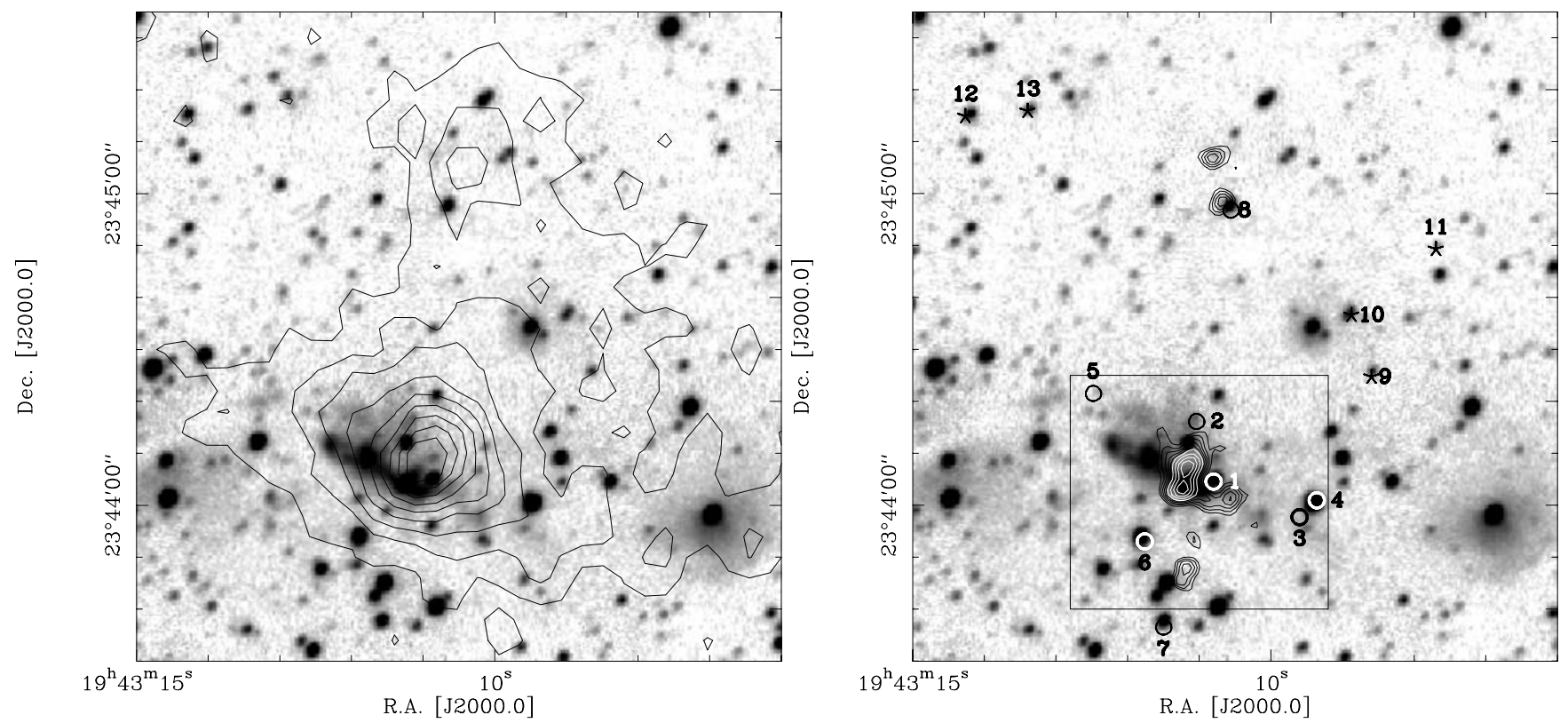

Fig. 1. Left: the contours present the large-scale $1.2 \mathrm{~mm}$ dust continuum emission from $10 \%$ to $90 \%$ (steps of $10 \%$ ) of the peak flux (Beuther et al. 2002a). The grey-scale shows the $K$-band image. Right: the contours show the PdBI $2.6 \mathrm{~mm}$ continuum dust cores (black: levels $3.6(1.2=$ $1 \sigma) 8.4 \mathrm{mJy} /$ beam, white: $9.6(2.4) 20 \mathrm{mJy} /$ beam) superposed on the $K$-band image. Circles and stars mark the X-ray sources presented in this paper. Sources with circles are most likely associated with the massive star-forming region, whereas the asterisks represent those that might be foreground objects. The box outlines the region presented in Fig. 4.

emission from intermediate-mass pre-main-sequence sources (Gregorio-Hetem et al. 1998). An example of hard X-ray emission from a Herbig Be star is MWC 297 (Hamaguchi et al. 2000). In a recent X-ray study of the Monoceros R2 molecular cloud, Kohno et al. (2002) detected X-ray emission for stars of all masses, in particular hard X-ray emission from high-mass pre-main-sequence or Zero-age-main-sequence stars. Based on these results, X-ray emission seems likely to be an ubiquitous phenomenon in the protostellar evolution of stars of all masses.

So far, X-ray studies in high-mass star-forming regions focused on more evolved massive star formation sites, while the very deeply embedded phase - and thus the youngest stage of stellar evolution - has not been detected at all. Results obtained in recent years by our group (Sridharan et al. 2002; Beuther et al. 2002a,b,d) and other groups studying massive star formation (e.g., Cesaroni et al. 1997; Zhang et al. 2002; Tan \& McKee 2002; Yorke 2002) support the hypothesis that massive stars form via disk accretion in a similar fashion as low-mass stars. Therefore, high-mass star-forming cores are promising candidate regions where in rather small spatial areas a number of sources could be hard X-ray emitters via the physical process of star-disk interactions (Hayashi et al. 1996; Montmerle et al. 2000). Necessary observational requirements are first of all sensitivity in the hard X-ray regime $(>2 \mathrm{keV})$, because only hard X-ray photons can penetrate high gas column densities. Additionally, high angular resolution is needed to resolve different sub-sources of the forming cluster. While ROSAT was not sensitive to hard X-ray photons, the spatial resolution of ASCA was not sufficient to study massive starforming regions in detail at their typical distances of a few kpc. The new-generation X-ray satellite telescopes Chandra and XMM-Newton comprise both features, being sensitive up to $10 \mathrm{keV}$, and having a spatial resolution of $0.5^{\prime \prime}$ (Chandra) and 15" (XMM-Newton), respectively. Especially Chandra is able to resolve many different sub-sources as impressively demonstrated in Orion by Garmire et al. (2000) and Feigelson et al. (2002).

Here, we present a Chandra X-ray study of the very young, massive and deeply embedded star-forming cluster IRAS $19410+2336$. IRAS $19410+2336$ is part of a large sample of 69 high-mass protostellar candidates which has been studied extensively in a series of papers during the last years (Sridharan et al. 2002; Beuther et al. 2002a-d). We assume the source to be located at its near kinematic distance of $2.1 \mathrm{kpc}$ (Sridharan et al. 2002), because the derived outflow parameters are unreasonably high for the far kinematic distance (Beuther et al. 2002b). At the distance of $2.1 \mathrm{kpc}$, its infrared derived luminosity is $10^{4} L_{\odot}$, and one observes two adjacent star-forming cores with masses of $840 M_{\odot}$ and $190 M_{\odot}$ (Sridharan et al. 2002; Beuther et al. 2002a). Each core drives a massive bipolar outflow in east-west direction (Beuther et al. 2002b). At the center of the southern massive core, a very compact and weak ( $\sim 1 \mathrm{mJy}$ ) cm wavelength source is detected, which coincides with $\mathrm{H}_{2} \mathrm{O}$ and Class II $\mathrm{CH}_{3} \mathrm{OH}$ maser emission (Beuther et al. 2002c). Figure 1 (left) gives an overview of the region of interest with the $1.2 \mathrm{~mm}$ dust continuum data (Beuther et al. 2002a) superposed on an infrared $K$-band image (Sect. 2.3). As the source is located in the Galactic plane, confusion due to foreground and background sources is expected and has to be disentangled by the different observations. We focus on the X-ray emission of this region and correlate the detected X-ray sources with high-resolution images in the $\mathrm{mm}$ and near-infrared regime. Section 2 describes the different observations we performed (X-ray, near-infrared and $\mathrm{mm}$ data), 
Table 1. Compilation of the parameters derived from the X-ray and 2MASS data: positions of the X-ray sources, number of counts per source, mean energy of detected photons, best fits of Raymond-Smith plasma temperatures $(k T[\mathrm{keV}]$ and $T[\mathrm{~K}])$ and derived X-ray luminosities $L_{\mathrm{X}}$ with error ranges. Colons mark less conclusive fits. Additionally, the column densities $N_{\mathrm{H}}$ (and error ranges) from the X-ray fits and the $2 \mathrm{MASS}$ derived extinctions $A_{\mathrm{v}}$ are presented.

\begin{tabular}{|c|c|c|c|c|c|c|c|c|c|}
\hline source & $\begin{array}{r}\text { RA } \\
(\mathrm{J} 2000.0)\end{array}$ & $\begin{array}{r}\text { Dec } \\
(\mathrm{J} 2000.0)\end{array}$ & $\begin{array}{r}\# \\
\text { [cts] }\end{array}$ & $\begin{array}{l}\text { mean } \\
{[\mathrm{keV}]}\end{array}$ & $\begin{array}{r}k T \\
{[\mathrm{keV}]}\end{array}$ & $\begin{array}{r}T \\
{\left[10^{7} \mathrm{~K}\right]}\end{array}$ & $\begin{array}{r}L_{\mathrm{X}} \\
{\left[10^{31} \mathrm{erg} \mathrm{s}^{-1}\right]}\end{array}$ & $\begin{array}{r}N_{\mathrm{H}} \\
{\left[10^{22} \mathrm{~cm}^{-2}\right]}\end{array}$ & $A_{\mathrm{v}}$ \\
\hline $\mathrm{X} 1$ & $19: 43: 10.8$ & $+23: 44: 04.6$ & 17 & 2.6 & $5.6[>4]$ & $6.5[>4.6]$ & $1.1[0.7-1.4]$ & $1.0[0.7-1.3]$ & 17: \\
\hline $\mathrm{X} 2$ & 19:43:11.0 & $+23: 44: 16.1$ & 10 & 3.9 & $9.6[>6]$ & $11.1[>7]$ & $2.1[1.5-2.8]$ & $5.9[4.5-8.0]$ & - \\
\hline $\mathrm{X} 3$ & 19:43:09.6 & $+23: 43: 57.7$ & 19 & 3.5 & 10: $[>6]$ & 11.6:[>7 ] & 2.4 [1.9-3.0] & $2.9[2.3-3.8]$ & - \\
\hline $\mathrm{X} 4$ & 19:43:09.4 & $+23: 44: 01.0$ & 17 & 2.5 & $3.2[2.2-5.3]$ & $3.7[2.6-6.2]$ & $1.2[0.8-1.7]$ & $1.1[0.9-1.4]$ & - \\
\hline X5 & $19: 43: 12.5$ & $+23: 44: 21.8$ & 9 & 5.3 & $9.3[>5]$ & $10.8[>5.8]$ & $2.6[1.6-3.5]$ & 10: $[>7.2]$ & - \\
\hline X6 & $19: 43: 11.8$ & $+23: 43: 53.0$ & 12 & 3.8 & $7.4[>5]$ & $8.6[>5.8]$ & $1.8[1.2-2.3]$ & $3.7[2.6-5.3]$ & 18 \\
\hline $\mathrm{X} 7$ & $19: 43: 11.5$ & $+23: 43: 36.5$ & 7 & 3.6 & $7.5[>4]$ & $8.7[>4.6]$ & $0.8[0.5-1.1]$ & $2.7[1.8-3.9]$ & 12 \\
\hline $\mathrm{X} 8$ & 19:43:10.6 & $+23: 44: 56.8$ & 12 & 2.2 & 1.4 [1.1-1.9] & $1.6[1.3-2.2]$ & $2.2[2.0-2.8]$ & $3.0[2.5-3.7]$ & 17 \\
\hline X9 & 19:43:08.6 & $+23: 44: 24.7$ & 9 & 2.8 & 1.2: & $1.4:$ & - & $0.3:$ & 12 \\
\hline $\mathrm{X} 10$ & 19:43:08.9 & $+23: 44: 36.6$ & 19 & 2.2 & 1.5: & 1.7: & - & 0.8: & 3 \\
\hline $\mathrm{X} 11$ & 19:43:07.7 & $+23: 44: 49.3$ & 7 & 1.2 & 1.0: & 1.2: & - & 0.2 : & - \\
\hline $\mathrm{X} 12$ & $19: 43: 14.3$ & $+23: 45: 14.9$ & 12 & 1.7 & $1.3[1.1-1.7]$ & $1.5[1.3-2.0]$ & - & $0.6[0.4-0.8]$ & 1 \\
\hline $\mathrm{X} 13$ & 19:43:13.4 & $+23: 45: 15.9$ & 13 & 1.2 & $1.3[1.1-1.5]$ & $1.5[1.3-1.7]$ & - & $0.3[0.2-0.4]$ & 2 \\
\hline
\end{tabular}

and in Sect. 3 we derive the main physical parameters of this star-forming region from our data (source detections, spectra, $\mathrm{X}$-ray luminosities, plasma temperatures and masses). Finally, Sect. 4 compiles our conclusions and puts the observational findings into a more general framework of high-mass star formation.

\section{Observations}

\subsection{Chandra $X$-ray observations and data reduction}

IRAS 19410+2336 was observed with the ACIS-S3 chip on board the X-ray telescope Chandra for $20 \mathrm{ksec}$ on October 15 2001. The ACIS-S3 aim-point was centered at the IRAS position RA 19:43:11.4, Dec 23:44:06.0. (J2000.0) that is coincident with the main $\mathrm{mm}$ emission peak. The field of interest shown in Fig. 1 is much smaller than the $\sim 8^{\prime} \times 8^{\prime}$ field of view of the S3 chip, and the FWHM of the point-spread-function of this region is approximately $0.5^{\prime \prime}$. The data were reduced with the CIAO 2.2 software package using the CALDB 2.10 database. Both packages are provided by the Chandra X-ray center ${ }^{1}$. The basic data product of our observation is the level 2 processed event list provided by the pipeline processing at the Chandra X-ray center.

Light curves were extracted for the whole S3 chip as well as just for the region presented in Fig. 1. No flares or enhancements due to low energy protons are observed. The whole observation time is usable as good time interval. The background emission is about 0.1 cts $^{\text {pixel }}{ }^{-1}$, but as one or two photons could be detected by background fluctuations anyway, we conservatively use $6 \mathrm{cts}$ as the detection threshold for an X-ray source in this field. A wavelet based source detection algorithm was applied using default parameters (Freeman et al. 2002), and 13 sources between 7 and $19 \mathrm{cts}$ are found in the field of interest (Table 1). Based on the $1.2 \mathrm{~mm}$ continuum map from the $30 \mathrm{~m}$ telescope - assuming optically thin dust

\footnotetext{
${ }^{1}$ http://asc.harvard.edu/ciao/download.html
}

emission -, we can estimate an average hydrogen column density of $N_{\mathrm{H}}=5 \times 10^{22} \mathrm{~cm}^{-2}$ of the molecular cloud (for details on the dust emission see Beuther et al. 2002a). A Raymond-Smith plasma model (Raymond \& Smith 1977) with a $3 \mathrm{keV}$ plasma, which is commonly used to model the X-ray emission of very young and embedded pre-main-sequence sources (Feigelson \& Montmerle 1999; Preibisch \& Zinnecker 2002), 6 cts correspond to an unabsorbed flux limit of $2 \times 10^{-14} \mathrm{erg} \mathrm{cm}^{-2} \mathrm{~s}^{-1}$. Based on the $\log N-\log S$ distribution of Giacconi et al. (2001), in a field as small as ours less than 0.1 extragalactic background sources are expected. The source positions in the field of interest are compared with 2MASS near-infrared data and should be correct within less than $1^{\prime \prime}$.

\subsection{Plateau de Bure $\mathrm{mm}$ observations}

We observed IRAS 19410+2336 in Summer 2001 with the Plateau de Bure Interferometer (PdBI) at $2.6 \mathrm{~mm}$ in the D (with 4 antennas) and $\mathrm{C}$ (with 5 antennas) configuration (Guilloteau et al. 1992). The simultaneously observed $1 \mathrm{~mm}$ data were only used for phase corrections because of the poor Summer weather conditions. The $3 \mathrm{~mm}$ receivers were tuned to $115.27 \mathrm{GHz}$ (USB) (centered at the ${ }^{12} \mathrm{CO} 1 \rightarrow 0$ line) with a sideband rejection of about $5 \mathrm{~dB}$. At this frequency, the typical SSB system temperature is 300 to $400 \mathrm{~K}$, and the phase noise was below $30^{\circ}$. Atmospheric phase correction based on the $1.3 \mathrm{~mm}$ total power was applied. For continuum measurements, we placed two $320 \mathrm{MHz}$ correlator units in the band to cover the largest possible bandwidths. In this paper we are focusing on the $2.6 \mathrm{~mm}$ continuum data, the CO line observations will be presented elsewhere. Temporal fluctuations of the amplitude and phase were calibrated with frequent observations of the quasars $1923+210$ and $2023+336$. The amplitude scale was derived from measurements of MWC349 and CRL618, and we estimate the final flux density accuracy to be $\sim 15 \%$. To cover both cores a mosaic of 10 fields was observed. The final beam size is $4.1^{\prime \prime} \times 3.6^{\prime \prime}\left(\mathrm{PA}-108^{\circ}\right)$. We obtain a $3 \sigma \mathrm{rms}$ of $\sim 5 \mathrm{mJy}$, 
which corresponds to a mass sensitivity limit of approximately $15 M_{\odot}$ assuming optically thin dust emission at $2.6 \mathrm{~mm}$ (see, e.g., Beuther et al. 2002a for the deviation of dust parameters from mm observations).

\subsection{Calar Alto near-infrared observations}

The near-infrared camera Omega Prime on the $3.5 \mathrm{~m}$ telescope on Calar Alto/Spain was used to obtain $K^{\prime}$ wide field images of the region around IRAS $19410+2336$ in June 2001. At a pixel scale of $0.4^{\prime \prime}$ pixel $^{-1}$, the $1024 \times 1024$ pixel array provides a field-of-view of $\sim 6.8^{\prime} \times 6.8^{\prime}$. A five position dither pattern was applied to image the field and to allow for a correction of array defects that were identified from well illuminated flat-field frames (for the dark pixels) and a dark frame (for the hot pixels). In each dither position, 15 individual exposures of $2 \mathrm{~s}$ were stacked into one frame of $30 \mathrm{~s}$ total integration time. For each position, the thermal background (sky) was computed by median combining the four frames resulting from the other dither positions. This sky frame was subtracted from the respective science frame, which also removes the bias level. The resulting frame was divided by a flat-field resulting from dome flats (the difference of a number of frames of the dome illuminated by a tungsten lamp and a number of frames taken without illumination). The images of the various dither positions were then registered and averaged into the final image. The total integration time in the center of the final image is $2.5 \mathrm{~min}$, which yields a detection limit ( $5 \sigma$ peak flux) for point sources of roughly $K^{\prime}=17.5$. The seeing-limited angular resolution in the final image is about $1.2^{\prime \prime}$. Because the observation was conducted at high air-masses, its photometry is rather poor. Thus, we used the Omega Prime data for identification only, and the photometry is taken from the 2MASS catalog.

\section{Results}

\subsection{Source detections and identification}

Figure 1 (right) shows that in the very young massive starforming region IRAS $19410+2336$ hard X-ray emission from several point sources is detected. No evidence for extended or diffuse emission is found within our detection limits (Sect. 2.1). At mm wavelengths, the two massive cores detected with the IRAM $30 \mathrm{~m}$ single-dish observations at an angular resolution of $11^{\prime \prime}$ (Beuther et al. 2002a) split up into many sub-sources when observed at higher spatial resolution (Fig. 1, right), confirming that a cluster of stars is forming. The interferometric data highlight the real massive cores, whereas the large-scale surrounding core emission is filtered out by the interferometric observing technique. A detailed analysis of the protocluster properties will be presented elsewhere. Here we are focusing on the spatial associations of X-ray, near-infrared and $\mathrm{mm}$ sources.

Thirteen X-ray sources are detected within the field of interest (Table 1). Remarkably, nearly all X-ray sources are, within the positional uncertainty, associated with near-infrared counterparts observed in the $K$-band (X3 and X5 only tentatively). Because the $K$-band extinction is approximately only $10 \%$ of the visual extinction, in the $K$-band we can observe more

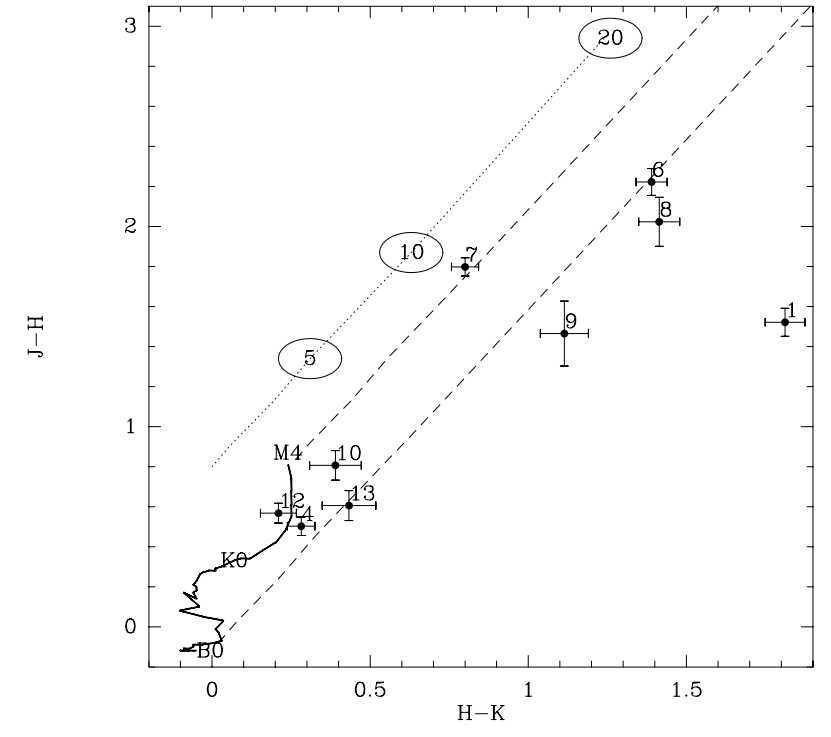

Fig. 2. Near-infrared color-color diagram of X-ray associated sources based on 2MASS data. The numbers correspond to the sources numbered also in Fig. 1. The full line presents main sequence colors with three labeled spectral star types (Ducati et al. 2001), and the dashed lines show the reddening band for main sequence colors (Rieke \& Lebofsky 1985). The dotted line gives the reddening scale with encircled visual extinctions ([mag]). Source X1 shows the strongest nearinfrared excess.

embedded regions of star-forming cluster than possible in the optical. We recall that the extinction in the soft X-ray regime is similar to the $K$-band extinction, and that it drops even further going to higher X-ray energies (Casanova et al. 1995; Ryter 1996). In contrast to the $K$-band counterparts, only 2 sources (X2 and X8) are found in the direct vicinity of $\mathrm{mm}$ dust cores that are tracing the coldest and deepest embedded regions where presumely the youngest sources are found. The whole region is also covered by the 2 Micron All Sky Survey $\left(2 \mathrm{MASS}^{2}\right)$ in the near-infrared bands $J, H$ and $K$. Except for sources X2, X3, X5 and X11, which are detected by our $K$-band Omega Prime observation but which are too faint to be detected by the 2MASS survey, all other X-ray sources are also 2MASS point sources. A color-color diagram helps to determine the characteristics of the associated near-infrared counterparts (Fig. 2). While a few of the near-infrared sources are located around the unreddened main sequence in the color-color diagram, many sources are reddened and show near-infrared excess, suggesting that they are pre-main-sequence objects surrounded by circumstellar material. This is expected in view of the early evolutionary stage, the large core masses and the high column densities of the region (Beuther et al. 2002a). Table 1 compiles reddening estimates of the X-ray near-infrared counterparts. The $A_{\mathrm{v}}$ of $\mathrm{X} 1$ is most uncertain because $\mathrm{X} 1$ has the largest near-infrared excess which makes the $A_{\mathrm{v}}$ determination more difficult. Using the reddening scale (Fig. 2), we can still estimate an approximate value of the visual extinction $A_{\mathrm{v}}$ of X1.

\footnotetext{
${ }^{2}$ For details see the 2MASS web-site at http://www.ipac. caltech.edu/2mass/releases/docs.html
} 


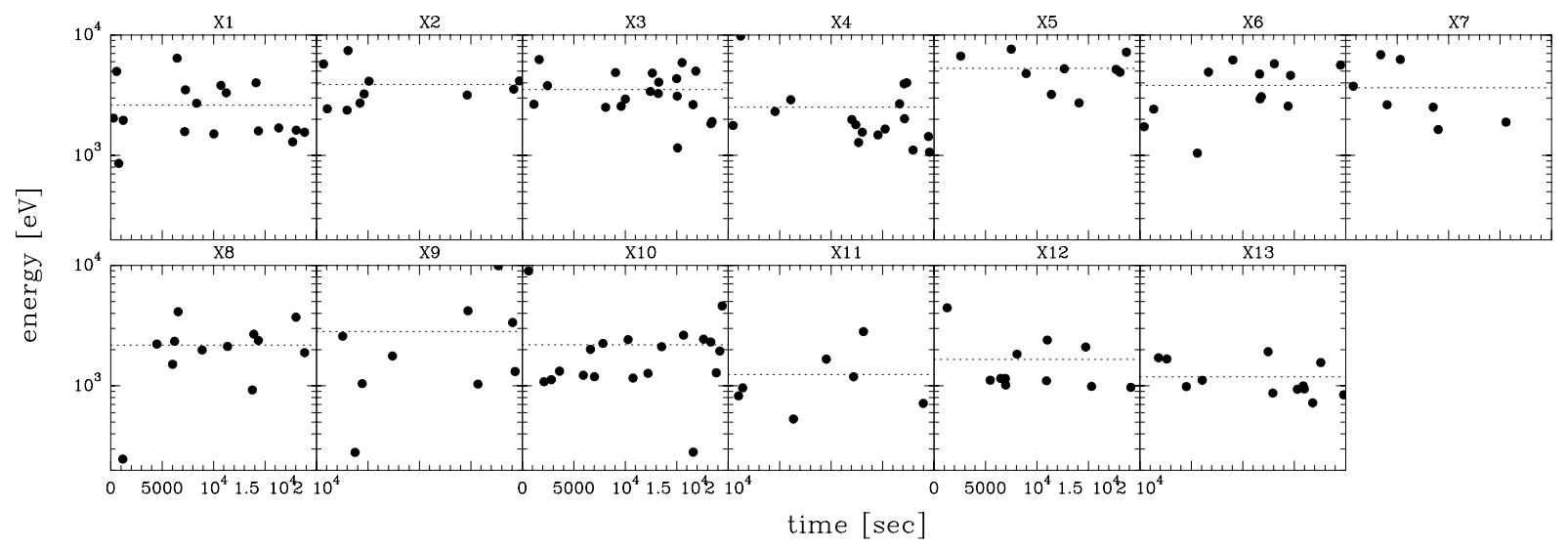

Fig. 3. Photon energy versus time for the 13 detected sources within the field of interest. The dotted lines mark the mean detected photon energy in each source (see also Table 1).

\subsection{Spectral information of the $X$-ray data}

\subsubsection{X-ray spectra and X-ray luminosities}

The number of photons per detected source ranges from 7 to 19 counts within the $20 \mathrm{ksec}$ observation time (Table 1). We note that we are dealing with low-number statistics, and this has to be taken into account when deriving the physical parameters of this region. Figure 3 presents the photon energies of the $13 \mathrm{X}$-ray sources versus time. As expected, we detect emission mainly between $2 \mathrm{keV}$ and $6 \mathrm{keV}$ in the near-infrared reddened and thus embedded sources, whereas the sources, that are near main sequence colors in the color-color diagram, emit more in the soft regime below $2 \mathrm{keV}$ (see also mean values in Fig. 3 and Table 1). The deficiency of soft photons $(<2 \mathrm{keV})$ in the direction of the embedded sources is most probably due to the high column densities that absorb the soft X-rays. The lack of photons above $6 \mathrm{keV}$ may be a real feature of the $\mathrm{X}$-ray sources, but it has also to be noted that the sensitivity of Chandra above $6 \mathrm{keV}$ decreases significantly. The effective collection area of Chandra at $8 \mathrm{keV}$ drops to less than a quarter of that at $4 \mathrm{keV}^{3}$. The fact that we detect hard $\mathrm{X}$-ray emission $(>3 \mathrm{keV})$ of the embedded sources fits in the general finding that the younger the sources are, the harder the X-ray emission is (e.g., Feigelson \& Montmerle 1999 or Sect. 1). The $\mathrm{X}$-ray spectra of X1 to X7 show all such hard X-ray signatures, which makes them likely to belong to the forming cluster. The distribution of photon energies cannot be explained by a lowtemperature-Raymond-Smith plasma of 1-2 keV (Raymond \& Smith 1977), usually used to fit the X-ray spectra of low-mass T Tauri stars (Feigelson \& Montmerle 1999), because the column densities $N_{\mathrm{H}}$, that are necessary to attenuate all the emission below $2 \mathrm{keV}\left(N_{\mathrm{H}}\right.$ a few times $\left.10^{23} \mathrm{~cm}^{-2}\right)$, would imply average $\mathrm{X}$-ray luminosities as high as $10^{34} \mathrm{erg} \mathrm{s}^{-1}$. This value exceeds the known luminosities of young stellar objects by orders of magnitude (Sect. 1), and it could hardly be reached even during strongest flaring events (e.g., Preibisch et al. 1993; Grosso et al. 1997; Tsuboi et al. 2000). Contrasting these hard spectra, X8 to X13 show softer spectra. While some of them

${ }^{3}$ See Chandra Proposers' Observatory Guide, 2000, http://cxc.harvard.edu might be unrelated objects, others could belong to the cluster but be in a more advanced state of evolution (for details see Sect. 3.4).

In order to derive estimates of the X-ray plasma temperatures and the X-ray luminosities, we performed a detailed spectral analysis. We extracted individual pulse-height spectra and built the corresponding redistribution matrices and ancillary response files for each source. We fitted the ungrouped pulse-height spectra with XSPEC, using a single-temperature thermal plasma model (model "raymond", Raymond \& Smith 1977) plus the absorption model "wabs". The plasma temperatures and column densities derived in these fits are compiled in Table 1. The fitting results were then used to integrate (in XSPEC) the model fluxes over the $0.2-10 \mathrm{keV}$ band and to compute the X-ray luminosities. In some cases the low number of detected photons allowed only to derive lower limits to the plasma temperature. We are fully aware that, given the rather low number of photons per source, the derived spectral parameters inherit a relatively large statistical uncertainty. Nevertheless, we believe that the fitting results contain valuable information, for the following three reasons:

(1) The spectral parameters derived in the fits allow a clear distinction of two different groups among the sources: sources $\mathrm{X} 1-\mathrm{X} 7$ show high plasma temperatures $(k T>3 \mathrm{keV})$ and high colum densities $\left(N_{\mathrm{H}}>10^{22} \mathrm{~cm}^{-2}\right)$; in contrast, the sources X8$\mathrm{X} 13$ show low plasma temperatures $(k T<1.5 \mathrm{keV})$ and (with the exception of X8) low colum densities $\left(N_{\mathrm{H}}<10^{22} \mathrm{~cm}^{-2}\right)$. These two groups exactly reflect the spatial distribution of the sources: $\mathrm{X} 1-\mathrm{X} 7$ are located inside the central region of the molecular clump, while X8-X13 lie outside the molecular clump (Fig. 1).

(2) The column densities derived in the spectral fits agree in nearly all cases rather well with expectations from the infrared colors of the sources (Table $1, A_{\mathrm{v}}=N_{\mathrm{H}} / 2 \times 10^{21}$ ).

(3) Getman et al. (2002) have performed fitting simulations of pulse-height spectra with low numbers of counts to estimate the statistical uncertainties of the fitting results. They found that even if the column densities and temperatures derived in the fits are subject to rather large uncertainties, the broad-band X-ray luminosities derived from the fitting results are quite reliable (statistical error $\leq 35 \%$ for spectra with 15 counts). 
Very likely, the X-ray emission of these objects originates from a hot thermal plasma typical for very young embedded objects (e.g., Feigelson \& Montmerle 1999; Preibisch \& Zinnecker 2002; Kohno et al. 2002). But as the number of photons and by that the spectroscopic information is small, other X-ray emission processes have to be taken into account, e.g., non-thermal power-law distributions as a signature of synchrotron emission, which has been observed in the radio regime by Reid et al. (1995) in the massive star-forming region $\mathrm{W} 3(\mathrm{OH})$. Therefore, we also estimate $\mathrm{X}$-ray luminosities with three different power-law distributions $S_{\mathrm{x}} \propto E^{-\alpha}(\alpha=$ $0.5,1,2)$ to derive an estimate for the influence of $\alpha$ on the total $\mathrm{X}$-ray luminosity (using the web-based PIMMS tool ${ }^{4}$ ). The derived luminosities vary by less than a factor 1.5 for the different $\alpha$. As hot plasmas are dominated by bremsstrahlung above $k T \sim 1 \mathrm{keV}$ (Fink \& Trümper 1982), which can also be approximated by non-thermal power-law distributions, the X-ray luminosities derived this way are close to the luminosities calculated using the Raymond-Smith plasma (they differ only a factor 2 at maximum).

We calculated $L_{\mathrm{x}}$ only for X1 to X8 (Table 1), because the other sources could be foreground or background objects, and thus no reliable distance is known. We find X-ray luminosities in the $10^{31} \mathrm{erg} \mathrm{s}^{-1}$ range. These values are high, and above or at the upper end of classical T Tauri X-ray luminosities, i.e., in the regime of younger Class I sources (Feigelson $\&$ Montmerle 1999) and/or more massive Herbig Ae/Be type objects (Zinnecker \& Preibisch 1994; Gregorio-Hetem et al. 1998; Hamaguchi et al. 2000). Unresolved binary systems could alter the results slightly, but not by orders of magnitude.

\subsubsection{Variability}

We do not see strong intensity flaring in our observations (Fig. 3), but sources X2, X3 and X4 show absence of detected photons in the time interval between $6 \times 10^{3}$ and $10^{4} \mathrm{~s}$, which indicates possible variability in these sources. The other sources show a rather constant count rate within the $20 \mathrm{ksec}$ of our observation. To quantify the possible variability, we performed a statistical test of the photon arrival times following the approach outlined by Preibisch \& Zinnecker (2002): given the number of detected photons in one source in the total integration time, we compute the mean count rate and specify the time period in which two photons are expected assuming a constant count rate. Then we determine the maximum number of photons $N_{\max }$ detected in this source within any time interval as long as the one derived for the two photons. The Poisson probability to find $N_{\max }$ or more counts due to statistical fluctuations in a period for which two photons are expected, is given by

$$
P=1-\sum_{k=0}^{N_{\max }-1} \mathrm{e}^{-2} \frac{2^{k}}{k !}
$$

The probability of variability (pov) is given by pov $=1-P$ (for details see, e.g., Bevington \& Robinson 1992). The pov values for $\mathrm{X} 3$ and $\mathrm{X} 4$ are only $68 \%$ and $85 \%$, giving just

\footnotetext{
${ }^{4}$ http://asc.harvard.edu/toolkit/pimms.jsp
}

marginal evidence for variability within our total observing time. Contrasting to that, pov equals $98.3 \%$ for $\mathrm{X} 2$, therefore this source is consistent with $\mathrm{X}$-ray variability.

\subsection{Mass estimates from the near-infrared data}

The 2MASS near-infrared $K$-band data provide a tool to estimate the masses of the infrared sources. Taking the observed $K$-band brightnesses $m_{K}$ at the given $K$-band extinction $\left(A_{K} \sim\right.$ $0.11 \times A_{\mathrm{v}}$ ) we get an estimate of the intrinsic brightness $M_{K}$ of the source assuming the distance $d=2 \mathrm{kpc}$ :

$m_{K}-M_{K}=5 \log (d)-5+A_{K}$.

Different pre-main-sequence tracks are discussed in the literature (e.g., D'Antona \& Mazzitelli 1994; Baraffe et al. 1998; Palla \& Stahler 1999; Siess et al. 2000; D'Antona et al. 2000). While some of them differ significantly at the low-mass end, they are better comparable going to masses $>1 M_{\odot}$ (Siess et al. 2000). Most of the calculated tracks do not cover stars more massive than $2.5 M_{\odot}$, but Palla \& Stahler (1999) calculate the pre-main-sequence evolution to $6 M_{\odot}$ and Siess et al. (2000) to $7 M_{\odot}$. At the high-mass end, which we are particularly interested in, both calculations agree well. The tracks of intermediate-mass stars $\left(>2 M_{\odot}\right)$ are following almost horizontal tracks of equal luminosity in the Hertzsprung-Russel diagram, and the luminosity is almost independent of the temperature. Thus, it is possible to estimate luminosities and by that masses from our $K$-band observations, but the data do not give any information about the age of the objects. Siess et al. (2000) stress that any age determination below $10^{6} \mathrm{yr}$ is highly uncertain. In the following, we use the tracks compiled by Palla \& Stahler (1999), but the results are similar when using the tracks of Siess et al. (2000).

Comparing the intrinsic $K$-band brightnesses $M_{K}$ with the theoretical tracks by Palla \& Stahler (1999), we can derive mass estimates for the near-infrared objects. We apply this procedure for the near-infrared sources corresponding to X1, X6, X7 and X8, because only those are 2MASS detections and likely belong to the forming massive clusters (Sect. 3.2). The premain-sequence tracks provided by Palla \& Stahler (1999) for stars up to $6 M_{\odot}$ do not comprise sources intrinsically brighter than $-1.6 \mathrm{mag}$ in the $K$-band. Sources more massive than $8 M_{\odot}$ do not have an optically visible pre-main-sequence phase, because they start nuclear burning already being deeply embedded in their natal cores (Palla \& Stahler 1993). Therefore, X6 and X7 could be more massive than $6 M_{\odot}$, but it has to be noted that the derived model brightnesses $M_{K}$ neglect luminosity due to the disk, i.e., heated dust in the disk, and accretion luminosity within the disk and between the disk and the protostar. Palla \& Stahler (1999) stress that the understanding of these processes is still inadequate for quantitative predictions, but as the near-infrared excess in $\mathrm{X} 6, \mathrm{X} 7$ and $\mathrm{X} 8$ is not very high (Fig. 2), to our judgment the additional disk luminosity in these sources is not the dominant effect. This is different for source $\mathrm{X} 1$ which has considerable infrared excess and makes $K$-band brightnesses difficult to be compared with theoretical predictions. But as stars below $2 M_{\odot}$ are at least 2 mag fainter in the theoretical predictions than the observed brightness of X1, 
Table 2. Near-infrared parameters of most likely cluster sources with 2MASS counterparts. Given are masses $\left(\leq 10 M_{\odot}\right)$, bolometric luminosities $L_{\mathrm{bol}}$, and the ratio $L_{\mathrm{X}} / L_{\mathrm{bol}}$.

\begin{tabular}{lrllrrrr}
\hline \hline src & $\begin{array}{r}m_{K} \\
{[\mathrm{mag}]}\end{array}$ & $A_{\mathrm{v}}$ & $A_{K}$ & $\begin{array}{r}M_{K} \\
{[\mathrm{mag}]}\end{array}$ & $\begin{array}{r}\text { mass } \\
{\left[M_{\odot}\right]}\end{array}$ & $\begin{array}{r}L_{\text {bol }} \\
{\left[L_{\odot}\right]}\end{array}$ & $\begin{array}{r}L_{\mathrm{X}} / L_{\text {bol }} \\
{\left[10^{-5}\right]}\end{array}$ \\
\hline $\mathrm{X} 1$ & 11.7 & 17 & 1.7 & -1.5 & $>2$ & $>10$ & $<70$ \\
$\mathrm{X} 6$ & 11.0 & 18 & 1.8 & -2.3 & $>6$ & - & - \\
$\mathrm{X} 7$ & 8.8 & 12 & 1.2 & -3.9 & $>6$ & - & - \\
$\mathrm{X} 8$ & 12.8 & 17 & 1.7 & -0.4 & $>3$ & $>30$ & $<2$ \\
\hline
\end{tabular}

$2 M_{\odot}$ can still be regarded as a tentative lower mass limit for this source. Real upper mass limits are given by the total bolometric luminosity of the whole cluster of $\sim 10^{4} L_{\odot}$ (Sridharan et al. 2002). A luminosity of $10^{4} L_{\odot}$ corresponds in the case of main sequence stars to a B1 star $\left(13 M_{\odot}\right)$. As a cluster of stars is forming in IRAS $19410+2336$, the mass distribution of the cluster members is likely to follow an initial mass function. In spite of their larger number, the luminosity-mass relation for low-mass stars is lower $\left(L \propto m^{2.8}\right.$ for $\left.m<1 M_{\odot}\right)$ than for high-mass stars $\left(L \propto m^{4}\right.$ for $1<m<30 M_{\odot}$, Schatzman $\&$ Praderie 1993), and still the major contribution of the total luminosity stems from the massive cluster members. For a detailed IMF discussion of the initial source sample see Sridharan et al. (2002). As the most massive object is likely the central $\mathrm{mm}$ source, which is not detected in X-ray emission, the X-ray detected sources are $\leq 10 M_{\odot}$. Additionally, the models give the approximate luminosity of the sources and we get an estimate of the $L_{\mathrm{X}} / L_{\text {bol }}$ ratio $\left(L_{\mathrm{X}}\right.$ is taken from Sect. 3.2 and Table 1). The parameters and results are listed in Table 2.

The derived masses are in the intermediate-mass regime of Herbig Ae/Be stars, clearly more massive than usual T Tauri or Class I sources. Zinnecker \& Preibisch (1994) observed $\mathrm{X}$-ray emission from Herbig $\mathrm{Ae} / \mathrm{Be}$ stars, and those sources showed rather soft spectra. Preibisch \& Zinnecker (1995) speculate that the observations could be explained by coronal emission due to a shear dynamo. As the spectral signatures of the sources we are studying here are significantly harder than those found in their sample (Sect. 3.2), we suggest that the $\mathrm{X}$-ray emission could be due to magnetic star-disk interactions as proposed for very young Class I sources (Montmerle et al. 2000). In that framework, it is possible that the sources observed in IRAS $19410+2336$ are precursors of the better studied Herbig Ae/Be stars. The possible $L_{\mathrm{X}} / L_{\mathrm{bol}}$ ratio-regime (Table 2) for two sources is at the upper boundary consistent with ratios found in very young low-mass objects $\left(\sim 2 \times 10^{-4}\right.$, e.g., Feigelson \& Montmerle 1999; Preibisch \& Zinnecker 2002), but at the lower end it is also consistent with the $L_{X} / L_{\text {bol }}$ ratios found in samples of Herbig Ae/Be stars (Zinnecker \& Preibisch 1994; Gregorio-Hetem et al. 1998). It could be argued that the X-ray emission is due to low-mass counterparts, but as the derived X-ray luminosities are significantly larger than typical values in the low-mass regime, most of the X-ray emission stems likely from the intermediate-mass sources.

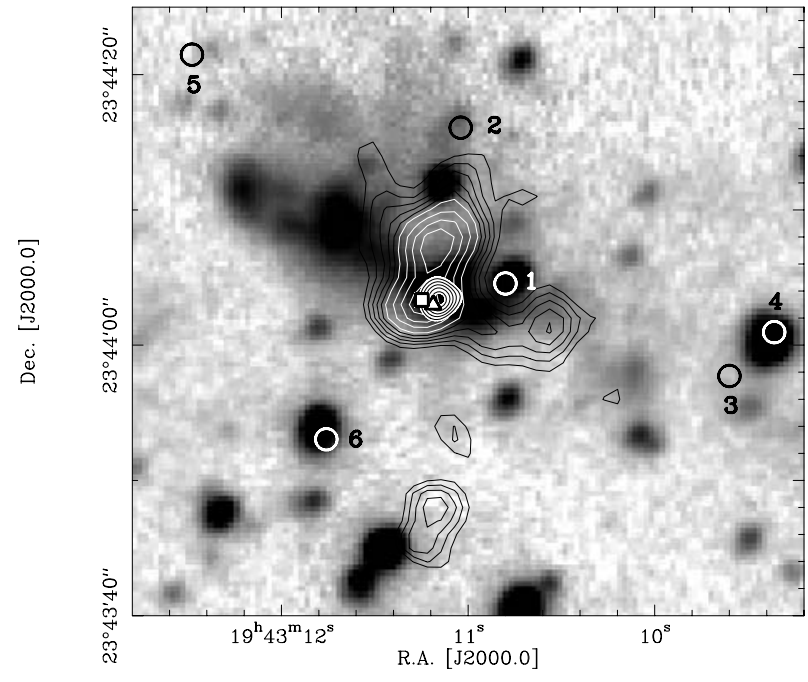

Fig. 4. Zoom into the center of the field presented in Fig. 1. Contours and symbols are the same as in Fig. 1. Additionally, the black contours on white ground right at the center show the $\mathrm{cm}$-peak, the triangle pinpoints the $\mathrm{H}_{2} \mathrm{O}$ maser position and the square the $\mathrm{CH}_{3} \mathrm{OH}$ maser positions (Minier et al. 2001; Beuther et al. 2002c).

\subsection{The $X$-ray sources in detail}

Source $\mathrm{X} 1$ and the main $\mathrm{mm}$ core: the most remarkable $\mathrm{X}$-ray source is $\mathrm{X} 1$, which does not only show strong reddening but also the largest infrared excess indicative embedded protostellar objects (Feigelson \& Montmerle 1999). As the infrared derived mass is $\geq 2 M_{\odot}$ but the X-ray spectrum harder than usual Herbig Ae/Be stars (Zinnecker \& Preibisch 1994), $\mathrm{X} 1$ is probably not a normal Herbig Ae/Be star but a precursor of such an object.

Source $\mathrm{X} 1$ is the $\mathrm{X}$-ray source nearest to the core center but not coincident with a mm core. Figure 4 shows that the main $\mathrm{mm}$ core coincides with a compact $\mathrm{cm}$ source as well as with $\mathrm{H}_{2} \mathrm{O}$ and Class II $\mathrm{CH}_{3} \mathrm{OH}$ maser emission (Minier et al. 2001; Beuther et al. 2002c), while the X-ray source is clearly offset and coincides with a near-infrared source $\sim 6^{\prime \prime}$ to the west. Thus, the main mm core is likely to contain the youngest and most massive object of the cluster, and we do not detect X-ray emission from this source, but only from another object nearby that might be slightly more evolved. Therefore, we do detect $\mathrm{X}$-ray emission from pre-main-sequence sources but not from the youngest and massive center of the cluster. Assuming optically thin dust emission, we estimate the core mass of the central source from the $2.6 \mathrm{~mm}$ continuum data to about $80 M_{\odot}$ and the $\mathrm{H}_{2}$ column density to $\approx 2 \times 10^{24} \mathrm{~cm}^{-2}$, corresponding to a visual extinction $A_{\mathrm{v}}$ of approximately 2000 (for details on mm dust calculations see Beuther et al. 2002a). Taking into account this visual extinction and assuming different Raymond-Smith plasma temperatures for the X-ray emission, we can calculate a range of upper limits for the X-ray luminosity of this source: a $3 \mathrm{keV}$ plasma temperature corresponds to an upper limit of $L_{\mathrm{X}}<9 \times 10^{34} \mathrm{erg} \mathrm{s}^{-1}$, while a temperature of $10 \mathrm{keV}$ implies $L_{\mathrm{X}}<4 \times 10^{34} \mathrm{erg} \mathrm{s}^{-1}$. A lower plasma temperature of $1 \mathrm{keV}$ results in an upper limit of $L_{\mathrm{X}}<14 \times 10^{36} \mathrm{erg} \mathrm{s}^{-1}$. However, it has to be noted that the possibility remains that there is no 
significant X-ray emission in the earliest stages of massive star formation.

Sources X2 and X5: source X2 is at the edge of the southern mm emission and associated with some faint $K$-band emission that might be a reflection nebula from the main sources to the south. As the $K$-band emission is weak, it is not detected by 2MASS. Being in the near vicinity of a $\mathrm{mm}$ core and showing a very hard X-ray spectrum, it is possible that X2 might be powered by an embedded protostellar object. It has to be noted that this source is the only one with observed X-ray variability. Source X5 is also too faint in the near-infrared to be detected by 2MASS, and it is difficult to decide whether the nearby faint $K$-band emission is a stellar feature or a reflection nebulae. But as X5 has the hardest X-ray spectrum of our sample, it is very likely a young protostellar object within the cluster of IRAS $19410+2336$.

Sources X3 and X4: these sources are both close to the same near-infrared source, but accurate astrometry indicates that X4 rather than X3 is associated with it (Fig. 4). Additionally, we find an optical counterpart for X4 in the Digitized Sky Survey provided by the Space Telescope Science Institute. Based on the X-ray spectrum, which is not as hard as the spectra of the other cluster members, and the optical and infrared data, we cannot distinguish whether $\mathrm{X} 4$ belongs to the cluster or is an unrelated foreground object. In contrast, X3 might be associated with more diffuse $K$-band emission (Fig. 4). As the hard $\mathrm{X}$-ray spectrum of X3 is indicating an early evolutionary stage, this source most likely is a very young pre-main-sequence object belonging to the cluster under investigation.

Sources X6 and X7: these sources also show hard X-ray spectra, and both are within the large-scale region of the southern massive core as seen in Fig. 1 (left), but no mm core corresponds to any of them. This is reflected in the color-color diagram, where they inhabit a reddened region without strong infrared excess. The infrared-derived mass estimates are not conclusive, but the data - X-ray and near-infrared - are consistent with precursors of Herbig Ae/Be stars of masses between $5 M_{\odot}$ and $10 M_{\odot}$.

Source X8: source $\mathrm{X} 8$ is associated with the strongest $\mathrm{mm}$ peak of the northern cluster as well as with a near-infrared and even an optical counterpart (as found in the Digitized Sky Survey). The 2MASS data show that the infrared source is reddened and has infrared excess, and we derive a lower mass limit of approximately $3.5 M_{\odot}$. The X-ray spectrum is softer than for the southern cluster members, thus it is possible that X8 is in a slightly more evolved state of evolution.

Sources $\mathrm{X} 9$ and $\mathrm{X} 10$ : the $\mathrm{X}$-ray spectrum of these sources is softer, but they are still reddened in the color-color diagram and $\mathrm{X} 9$ even shows infrared excess. It is likely that they are also young stellar objects, but we are not able to determine whether they belong to the cluster or whether they are foreground objects.

Sources $\mathrm{X} 11, \mathrm{X} 12$ and $\mathrm{X} 13$ : these sources also have a rather soft spectrum, and the color-color diagram locates them on or near the unreddened main sequence (X11 is too weak to be a 2MASS detection). As they are also spatially offset from the large-scale mm core, we regard them as not associated with the massive star-forming region.

\section{Conclusions}

Hard X-ray emission from a number of point sources is detected in a young, massive and embedded star-forming cluster in a very early stage of evolution. We did not detect $\mathrm{X}$-ray emission from the most massive and central object (upper limit $L_{\mathrm{X}}<9 \times 10^{34} \mathrm{erg} \mathrm{s}^{-1}$ for $k T=3 \mathrm{keV}$ ) but from a few sources in its vicinity. Typical X-ray properties of highmass main-sequence stars $\left(M_{\star} \geq 8 M_{\odot}\right)$, where the emission originates from internal shocks in the radiation-driven stellar winds, are $L_{\mathrm{X}} / L_{\mathrm{bol}} \sim 10^{-7}$, soft X-ray spectra with typical temperatures of $k T \lesssim 0.5 \mathrm{keV}$ and very little variability (Berghöfer et al. 1997). The X-ray properties we find for $\mathrm{X} 1$ to $\mathrm{X} 7$ are clearly different, they show hard X-ray emission $(k T \geq 3 \mathrm{keV})$ and most of them also near-infrared excess, very similar to those observed for extremely young embedded objects (Class I protostars), which have typical plasma temperatures of $k T \sim 5-10 \mathrm{keV}$ (Feigelson \& Montmerle 1999; Imanishi et al. 2001). Combining infrared data with premain-sequence evolutionary tracks (Palla \& Stahler 1999), it is possible to estimate the approximate masses of some of the hard X-ray sources. Those estimates indicate that they are in the intermediate-mass regime of Herbig Ae/Be objects. Taking additionally into account the harder X-ray spectra compared with other Herbig Ae/Be studies, it is likely that the X-ray sources in IRAS $19410+2336$ are even precursors of Herbig Ae/Be stars. The emitted X-ray photons with energies mostly above $2 \mathrm{keV}$ indicate plasma temperatures $>10^{7} \mathrm{~K}$ and $\mathrm{X}$-ray luminosities around a few times $10^{31} \mathrm{erg} \mathrm{s}^{-1}$. The latter values are well within the regime of Class I low-mass protostars (Feigelson \& Montmerle 1999), but they are also consistent with the results obtained for Herbig Ae/Be stars (Zinnecker \& Preibisch 1994). Thus, some of the objects are probably very young intermediate-mass pre-main-sequence sources, whereas other sources could also be low-mass Class I or T Tauri stars. The emission of one of the sources is consistent with X-ray variability.

In spite of the observation of hard X-ray emission in the weak-lined T Tauri star V773 Tau (Tsuboi et al. 1998), where the disk has already been dissipated to a large degree, it is unlikely that the hard X-ray spectra observed in younger class I sources are due to enhanced solar-type magnetic activity. Therefore, it is proposed that the hard X-ray emission, which is more often observed in class I sources than in weak-lined $\mathrm{T}$ Tauri stars, is produced by magnetic reconnection effects between the protostars and their accretion disks (Hayashi et al. 1996; Feigelson \& Montmerle 1999; 
Montmerle et al. 2000). As the X-ray spectra of the intermediate-mass objects in IRAS $19410+2336$ exhibit very similar signatures to such low-mass sources, our results are consistent with disks being present in intermediate-mass star formation as well.

For a better understanding of the nature of the underlying $\mathrm{X}$-ray powering sources much work has to be done in the future. Deeper X-ray and near-infrared images will help to set stronger constraints on the physical properties of the sources: it will be necessary to obtain sensitive X-ray spectra to determine better the absorbing $N_{\mathrm{H}}$ column densities and plasma parameters. It is also of great interest to further investigate the properties of the central and deepest embedded object, which means lowering the detection limits. Furthermore, the variability of the X-ray sources in very young massive star-forming regions is not known so far. Therefore, several approaches should be followed in the years coming: deep Chandra observations of the source of interest will disclose variabilities and faint emission of the central object. Additionally, a sample of similar sources has to be identified, because only a statistical analysis of several young high-mass star-forming regions can build a solid picture of the relevant physical processes. As high spatial resolution is essential for many of this studies, Chandra is a very promising choice. But considering the higher sensitivity of XMM-Newton, it might be possible to study grating X-ray spectra of the brightest sources of the sample of clusters studied then. On the near-infrared side, we suggest to get deeper images in the $J, H$ and $K$ bands to improve the mass estimates of the X-ray emitting sources, and near-infrared spectroscopy might help classifying the types of stars (Hanson et al. 2002).

To summarize, X-ray studies of young massive starforming regions are just in its infancy, and the next years with the space telescopes Chandra and XMM-Newton will bring many new insights in that research area. We also like to stress that multi-frequency studies over a wide range of bands are extremely promising approaches for the understanding of the physical processes forming massive stars.

Acknowledgements. We thank an anonymous referee for very helpful and detailed comments on the paper. This publication makes use of data products from the Two Micron All Sky Survey, which is a joint project of the University of Massachusetts and the Infrared Processing and Analysis Center, funded by the National Aeronautics and Space Administration and the National Science Foundation. We also used data from the Digitized Sky Survey as provided by the Space Telescope Science Institute.

\section{References}

Baraffe, I., Chabrier, G., Allard, F., \& Hauschildt, P. H. 1998, A\&A, 337, 403

Beuther, H., Sridharan, T. K., Schilke, P., et al. 2002a, ApJ, 566, 945

Beuther, H., Schilke, P., Sridharan, T. K., et al. 2002b, A\&A, 383, 892

Beuther, H., Walsh, A., Schilke, P., et al. 2002c, A\&A, 390, 289

Beuther, H., Schilke, P., Gueth, F., et al. 2002d, A\&A, 387, 931

Berghöfer, T. W., Schmitt, J. H. M. M., Danner, R., \& Cassinelli, J. P. 1997, A\&A, 322, 167

Bevington, P. R., \& Robinson, D. K. 1992, 2nd edition of Data reduction and error analysis for the physical sciences (McGraw-Hill)
Casanova, S., Montmerle, T., Feigelson, E. D., \& André, P. 1995, ApJ, 439, 752

Cesaroni, R., Felli, M., Testi, L., Walmley, C. M., \& Olmi, L. 1997, A\&A, 325, 725

Churchwell, E. 2001, Proc. of the IAU General Assembly 2000 in Manchester, ed. P. S. Conti, \& E. B. Churchwell

D’Antona, F., \& Mazzitelli, I. 1994, ApJS, 90, 467

D’Antona, F., Ventura, P., \& Mazzitelli, I. 2000, ApJ, 543, L77

Ducati, J. R., Bevilacqua, C. M., Remold, S., \& Ribiero, D. 2001, ApJ, 558,309

Feigelson, E., \& Montmerle, T. 1999, ARA\&A, 37, 363

Feigelson, E. D., Broos, P., Gaffney, J. A., et al. 2002, ApJ, in press

Fink, H. H., \& Trümper, J. 1982, in Landolt-Börnstein, Group VI, vol. 2, subvol. C, 38

Freeman, P. E., Kashyap, V., Rosner, R., \& Lamb, D. Q. 2002, ApJ, in press

Frerking, M., Langer, L., \& Wilson, R. 1982, ApJ, 262, 590

Garmire, G., Feigelson, E. D., Broos, P., et al. 2000, AJ, 120, 1426

Getman, K. V., Feigelson, E. D., Townsley, L., et al. 2002, ApJ, in press [astro-ph/0204252]

Giacconi, R., Rosati, P., Tozzi, P., et al. 2001, ApJ, 551, 624

Gregorio-Hetem, J., Montmerle, T., Casanova, S., \& Feigelson, E. D. 1998, A\&A, 331, 193

Grosso, N., Montmerle, T., Feigelson, E. D., et al. 1997, Nature, 387, 56

Guilloteau, S., Delannoy, J., Downes, D., et al. 1992, A\&A, 323, 943

Hanson, M. M., Luhman, K. L., \& Rieke, G. H. 2002, ApJS, 138, 35

Hayashi, M. R., Shibata, K., \& Matsumoto, R. 1996, ApJ, 468, L37

Hamaguchi, K., Terada, H., Bamba, A., \& Koyama, K. 2000, ApJ, 532,1111

Hofner, P., \& Churchwell, E. 1997, ApJ, 486, L39

Imanishi, K., Koyama, K., \& Tsuboi, Y. 2001, ApJ, 557, 747

Kohno, M., Koyama, K., \& Hamaguchi, K. 2002, ApJ, 567, 423

Minier, V., Conway, A. G., \& Booth, R. S. 2001, A\&A, 369, 278

Montmerle, T., Grosso, N., Tsuboi, Y., \& Koyama, K. 2000, ApJ, 532, 1097

Palla, F., \& Stahler, S. W. 1993, ApJ, 418, 414

Palla, F., \& Stahler, S. W. 1999, ApJ, 525, 772

Preibisch, T., Zinnecker, H., \& Schmitt, J. H. M. M. 1993, A\&A, L33

Preibisch, T., \& Zinnecker, H. 1995, in MPE report 263, ed. H. U. Zimmermann, J. Trümper, \& H. W. Yorke, 17

Preibisch, T., \& Zinnecker, H. 2002, AJ, 123, 1613

Ramond, J. C., \& Smith, B. W. 1977, ApJS, 35, 419

Reid, M. J., Argon, A. L., Masson, C. R., Menten, K. M., \& Moran, J. M. 1995, ApJ, 443, 238

Rieke, G. H., \& Lebofsky, M. J. 1985, ApJ, 288, 618

Ryter, C. E. 1996, Ap\&SS, 236, 285

Siess, L., Dufour, E., \& Forestini, M. 2000, A\&A, 358, 593

Schatzman, E., \& Praderie, F. 1993, The Stars (Springer Verlag)

Sridharan, T. K., Beuther, H., Schilke, P., Menten, K., \& Wyrowski, F. 2002, ApJ, 566, 931

Tan, J., \& McKee, C. 2002, Proc. of The earliest stages of massive star formation, to be published in ASP Conf. Ser., ed. P. Crowther

Tsuboi, Y., Koyama, K., Murakami, H., et al. 1998, ApJ, 503, 984

Tsuboi, Y., Imanishi, K., Koyama, K., Grosso, N., \& Montmerle, T. 2000, ApJ, 532, 1089

Tsuboi, Y., Koyama, K., Hamaguchi, K., et al. 2001, ApJ, 554, 734

Tsujimoto, M., Koyama, K., Tsuboi, Y., et al. 2002, ApJ, 573, 270

Yorke, H. 2002 , Proc. of The earliest stages of massive star formation, to be published in ASP Conf. Ser., ed. P. Crowther

Zhang, Q., Hunter, T. R., Sridharan, T. K., \& Ho, P. T. P. 2002, ApJ, in press

Zinnecker, H., \& Preibisch, T. 1994, A\&A, 292, 152 